Reprod. Nutr. Dévelop., 1980, 20 (4 B), 1237-1245.

\title{
Rôle de la muqueuse duodénale dans l'adaptation de l'a-amylase pancréatique au régime alimentaire chez le Porc
}

\author{
par C. SIMOES NUNES, T. CORRING \\ avec la collaboration technique de Anne-Marie GUEUGNEAU, \\ Georgette FREDOU, A. ROGER et A. VAN HECKE
}

Laboratoire de Physiologie de la Nutrition

I. N. R. A. 78350 jouy en Josos, France

Summary. The role of the duodenal mucoso in the adaptation of pancreatic $\alpha$-amylase to the diet in the pig.

Exocrine pancreatic secretion was studied in the conscious, fistulated pig before and after (i) intravenous glucose injection and (ii) intravenous injections of intestinal mucosae obtained from pigs adapted to a high-starch diet and taken 30 and 60 min after the beginning of a meal of the same diet. Within 30 min after it was injected, the glucose inhibited pancreatic juice volume $(-45.4$ p. $100 ; P<0.001)$, total protein output $(-54.2$ p. 100 ; $P<0.001)$ and specific amylase activity $(-15.8$ p. $100 ; P<0.001)$. Specific chymotrypsin activity increased $(+5$ p. $100 ; P<0.1)$, while that of lipase was not affected. On the contrary, the intravenous injection of intestinal mucosae increased pancreatic juice volume $(>49$ p. $100 ; \mathrm{P}<0.01)$, total protein output $(>55$ p. $100 ; \mathrm{P}<0.01)$ and specific amylase activity ( $>33$ p. $100 ; P<0.001)$, whilst specific lipase activity was inhibited $(<8$ p. 100 ; $P<0.01)$. Specific chymotrypsin activity was unmodified. It is suggested that the duodenal mucosa may play a role in pancreatic enzyme adaptation to the diet; the possible mechanisms involved in this process are discussed.

\section{Introduction.}

La nature des mécanismes impliqués dans l'adaptation enzymatique de la sécrétion pancréatique exocrine aux constituants du régime alimentaire est encore ignorée. Selon plusieurs auteurs, les produits d'hydrolyse digestive des glucides, lipides et protéines de la ration seraient impliqués dans ces mécanismes (Ben Abdeljlil et Desnuelle, 1964 ; Snook, 1971 ; Johnson, Hurwitz et Kretchmer, 1977).

Selon une hypothèse récemment émise, les variations de la taille du «pool» intestinal des produits d'hydrolyse seraient le point de départ de l'information qui induit les medifications spécifiques adaptatives de la sécrétion exocrine du pancréas (Corring, 1977). Cette hypothèse a été en partie vérifiée en ce qui concerne l'adaptation à la teneur en glucides du régime alimentaire (Simoes Nunes et Corring, 1979a). Il a été montré que la sécrétion pancréatique du Porc est capable de répondre très rapidement $(2 h)$ à une augmentation de la quantité de glucose présente dans le duo- 
dénum par un accroissement de l'activité spécifique de l'amylase. Au terme de cette étude, il a été suggéré que le glucose est le point de départ de l'information au pancréas induisant l'adaptation de l' $\alpha$-amylase pancréatique. Mais la question que l'on peut se poser à présent est la suivante : comment est transmise cette information? Le travail reporté ici a pour but de répondre à cette question.

\section{Matériel et méthodes.}

Animaux ef alimentation. - Sept porcs de race Large White, âgés de 90 à 120 jours d'un poids vif moyen de $46,4 \pm 2,1 \mathrm{~kg}$ ont été utilisés.

Deux d'entre eux ont été alimentés ad libitum pendant 10 jours avec un régime riche en amidon (60 p. 100) et ont été utilisés comme «donneurs » de muqueuse duodénale. Les cinq autres ont été munis de fistules chroniques du conduit pancréatique, du duodénum (Corring, Aumaitre et Rérat, 1972) et de la veine jugulaire externe droite ef ont été utilisés comme animaux « receveurs». Ils ont été nourris avec un régime équilibré. La composition des deux régimes a été antérieurement décrite (Simoes Nunes et Corring, 1979a : régime riche en amidon = régime $A$; régime équilibré $=$ régime $C$ ).

Après l'intervention chirurgicale et pendant toute la durée de l'expérimentation, les animaux ont été logés en cages individuelles placées dans une salle isolée pour éviter de les soumettre à d'autres stimulations que celles envisagées dans le schéma expérimental.

Les essais n'ont débuté que 8 à 10 jours après l'intervention chirurgicale.

Prélèvements de muqueuse duodénale. - Après 10 jours d'adaptation au régime riche en amidon, les animaux «donneurs» ont été abattus respectivement 30 et 60 min après le début de la prise d'un repas du même régime. Immédiatement après abattage une portion de l'intestin grêle représentant les 80 à $100 \mathrm{~cm}$ post-pyloriques, a été prélevée, rincée avec une solution de $\mathrm{NaCl}$ 0,9 p. 100 glacée. La muqueuse recueillie par grattage a été aussitôt broyée à froid à l'aide d'un microbroyeur (1) dans 4 à 5 fois son poids d'eau distillée, congelée à $-80^{\circ} \mathrm{C}$ et lyophilisée.

Schéma expérimental. - Le principe de l'expérience consiste à étudier sur les porcs fistulés («receveurs ») la composition enzymatique, le volume et les protéines totales du suc pancréatique recueilli après injections intraveineuses des lyophilisats de muqueuse duodénale prélevée chez les animaux «donneurs»adaptés au régime riche en amidon.

Les essais ont été effectués après un jeûne de $18 \mathrm{~h}$, l'animal fistulé ayant libre accès à l'eau de boisson. Chaque porc (« receveur ») a été soumis aux interventions suivantes :

- injections intraveineuses par l'intermédiaire du cathéter jugulaire de $100 \mathrm{ml}$ de solutions de chlorure de sodium 0,9 p. 100 et de glucose 15 p. 100 ;

- injections intraveineuses des lyophilisats de muqueuse duodénale provenant des animaux adaptés au régime riche en amidon à raison de $2 \mathrm{mg}$ (correspondant à $8 \mathrm{mg}$ de muqueuse fraîche) par $\mathrm{kg}$ de poids vif dans $100 \mathrm{ml}$ d'eau distillée.

(1) Omni-Mixer, Sorval, Dupont de Nemours S. A. France, Orsay. 
Toutes les injections ont été pratiquées lentement $( \pm 3 \mathrm{~min}$ ) et ont été précédées et suivies d'une injection de $20 \mathrm{ml}$ de solution de chlorure de sodium $0,9 \mathrm{p} .100$ héparinée (500 unités internationales d'héparine (1) par $\mathrm{ml}$ ).

Collecte de suc pancréatique et défermination des activités enzymatiques. - Les modalités de collecte de suc pancréatique ont été décrites précédemment (Simoes Nunes ef Corring, 1979a). Pour chacun des essais cinq échantillons d'environ $10 \mathrm{ml}$ chacun ont été prélevés et analysés : le premier correspondant aux 30 min qui ont précédé l'injection et les quatre autres, un toutes les $30 \mathrm{~min}$, pendant les deux heures qui l'ont suivie.

Dans chacun des échantillons de suc pancréatique, il a été procédé aux déterminations des protéines totales et des activités enzymatiques de la chymotrypsine, de l'amylase et de la lipase selon les méthodes citées précédemment (Simoes Nunes et Corring, 1979a). Toutes les activités enzymatiques sont présentées en activités spécifiques (par $\mathrm{mg}$ de protéines totales). Par ailleurs, dans chacun des lyophilisats de muqueuse duodénale il a été procédé à la détermination de la teneur en glucose (Huggett et Nixon, 1957).

Dans toutes les expériences réalisées, la sécrétion pancréatique a été retournée à l'animal. On a ainsi éliminé la possibilité de stimulation de cette sécrétion causée par l'absence d'enzymes pancréatiques, notamment des protéases dans la lumière intestinale (Corring, 1974).

\section{Résultats.}

En raison des importantes variations individuelles les données obtenues pendant les $30 \mathrm{~min}$ et les $120 \mathrm{~min}$ (moyenne de 4 valeurs) après injections sont exprimées en pourcentage de la valeur déterminée au cours des $30 \mathrm{~min}$ qui les ont précédées. Par ailleurs, les effets de l'injection intraveineuse de glucose et des lyophilisats ont été comparés à ceux de l'injection intraveineuse de $\mathrm{NaCl} 0,9$ p. 100.

Toutes les données ont été interprétées statistiquement (test « $t$ » de Student).

Effets sur le volume de la sécrétion pancréatique (tabl. 1). - L'injection intraveineuse de la solution de glucose 15 p. 100 entraîne une diminution très significative du volume de suc pancréatique excrété. Cet effet est persistant, car il est encore évident 120 min après injection, bien que tendant vers un rétablissement de sa valeur.

L'injection intraveineuse des lyophilisats de muqueuse provoque une augmentation de la quantité de suc excrété, le volume étant toujours supérieur de plus de $56 \mathrm{p}$. 100 à celui écoulé après injection intraveineuse de $\mathrm{NaCl} 0,9$ p. 100. Par ailleurs, aucune différence n'apparaît dans l'effet des lyophilisats des muqueuses comparés entre eux.

Effets sur les protéines totales excrétées (tabl. 2). - Le glucose en solution hypertonique provoque, au cours des 30 min suivant son injection intraveineuse, une diminution très importante des protéines excrétées dans le suc pancréatique $(-50$ p. 100 de la quantité excrétée après injection de $\mathrm{NaCl} 0,9$ p. 100). La quantité de protéines

(1) Héparine, Laboratoire Roche, Neuilly-sur-Seine. 


\section{TABLEAU 1}

Effets sur le volume de suc pancréatique excrété des injections intraveineuses de $\mathrm{NaCl}$, de glucose el des lyophilisats de muqueuses prélevées sur les onimaux adaptés

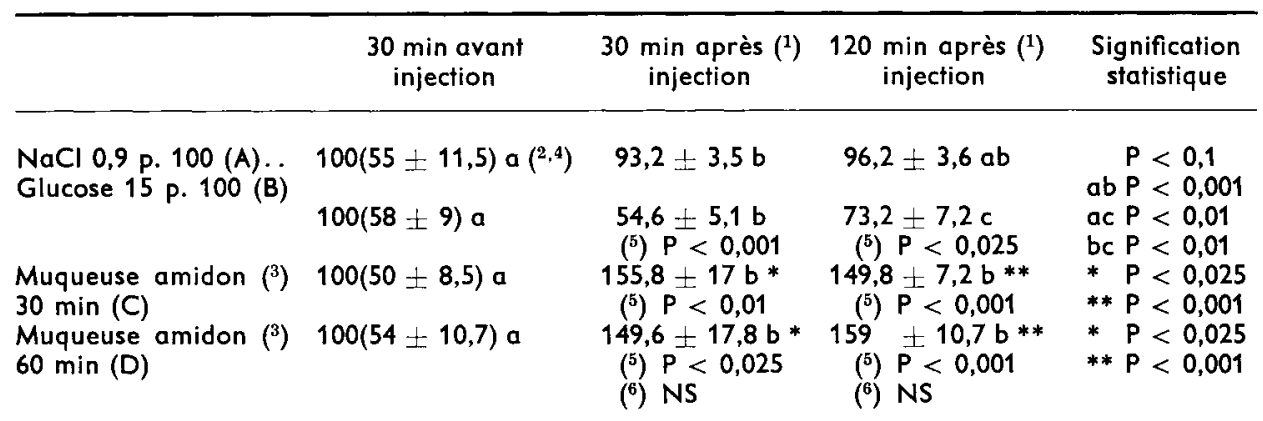

Volume $(\mathrm{ml})$ excrété par périodes de $30 \mathrm{~min}$ avant et après injections de $\mathrm{NaCl} 0,9$ p. 100 (A), de glucose 15 p. $100(B)$ et des lyophilisats de muqueuses intestinales d'animaux adaptés à un régime riche en amidon et prélevées $30 \mathrm{~min}$ (C) et $60 \mathrm{~min}$ (D) après le début de la prise d'un repas.

$\left.{ }^{(}\right)$Valeurs exprimées en pourcentage des valeurs de base obtenues au cours des 30 min avant injection. $\left({ }^{2}\right)$ Moyenne \pm écart type des valeurs réelles prises comme base $100 .\left({ }^{3}\right) 2$ mg de lyophilisat par $\mathrm{kg}$ de poids vif. $\left({ }^{4}\right)$ Les moyennes non suivies de la même lettre $(a, b, \ldots)$ sont significativement différentes au seuil indiqué dans la colonne «signification statistique ». (5) Comparaison de $A$ avec $B$, $C$ et D. $\left(^{6}\right)$ Comparaison de C avec D.

\section{TABLEAU 2}

Effets sur les protéines totales excrétées dans le suc pancréatique des injections intraveineuses de $\mathrm{NaCl}$, de glucose et des lyophilisats de muqueuses prélevées sur les animaux adaptés

\begin{tabular}{|c|c|c|c|c|}
\hline & $\begin{array}{l}30 \text { min avant } \\
\text { injection }\end{array}$ & $\underset{\text { injection }}{30}$ min après $\left({ }^{1}\right)$ & $\begin{array}{c}120 \text { min après }\left(^{1}\right) \\
\text { injection }\end{array}$ & $\begin{array}{l}\text { Signification } \\
\text { statistique }\end{array}$ \\
\hline $\begin{array}{l}\mathrm{NaCl} 0,9 \text { p. } 100(\mathrm{~A}) \\
\text { Glucose } 15 \text { p. } 100(\mathrm{~B}) \\
\text { Muqueuse amidon }\left({ }^{3}\right) \\
30 \text { min (C) } \\
\text { Muqueuse amidon }\left({ }^{3}\right) \\
60 \text { min (D) }\end{array}$ & $\begin{array}{l}100(111,5 \pm 20,8) a\left(^{2,4}\right) \\
100(177,1 \pm 56,8) a \\
100(110,7 \pm 23,5) a \\
100(120,8 \pm 28,5) a\end{array}$ & $\begin{array}{l}93,8 \pm 2 b \\
45,8+3 b \\
\left({ }^{5}\right) \mathrm{P}<0,001 \\
155,4 \pm 9,4 b * \\
\left(^{5}\right) \mathrm{P}<0,001 \\
70,8 \pm 22,3 \mathrm{~b} * \\
\left(^{5}\right) \mathrm{P} \pm 0,01 \\
\text { (6) NS }\end{array}$ & $\begin{array}{c}96,4 \pm 2,4 \mathrm{ab} \\
58,4 \pm 5,9 \mathrm{c} \\
\left(^{5}\right) \mathrm{P}<0,001 \\
174,2 \pm 37 \mathrm{~b} * * \\
\left(^{5}\right) \mathrm{P}<0,1 \\
176,6 \pm 10,5 \mathrm{~b} * * \\
\text { (5) } \mathrm{P}<0,001 \\
\text { (6) NS }\end{array}$ & $\begin{aligned} \mathrm{P} & <0,025 \\
\mathrm{abc} P & <0,001 \\
\mathrm{bc} \mathrm{P} & <0,01 \\
* \mathrm{P} & <0,001 \\
* * \mathrm{P} & <0,01 \\
* \mathrm{P} & <0,025 \\
* * \mathrm{P} & <0,001\end{aligned}$ \\
\hline
\end{tabular}

Protéines totales $(\mathrm{mg})$ excrétées (cf. légende tabl. 1).

excrétées subit une légère augmentation dans la phase finale de la période d'étude, mais reste en moyenne toujours très inférieure à celle obtenue avec $\mathrm{NaCl} 0,9$ p. 100 .

L'injection des lyophilisats de muqueuse entraîne une augmentation très significative des protéines totales excrétées, la quantité ayant tendance à être un peu plus importante après injection du lyophilisat de muqueuse prélevée $60 \mathrm{~min}$ après le début de la prise du repas. Par ailleurs, l'effet des deux muqueuses persiste pendant toute la durée de l'étude. 
Effets sur les activités enzymatiques spécifiques. - L'activité spécifique de l'amylase (tabl. 3) diminue significativement après injection de la solution de glucose 15 p. 100. Son évolution dans le temps est semblable à celle observée après injection de la solution isotonique de $\mathrm{NaCl}$, mais sa valeur est toujours bien moins importante.

TABLEAU 3

Effets de l'activité spécifique de l'amylase dans le suc pancréatique des injections intraveineuses de $\mathrm{NaCl}$, de glucose et des lyophisats de muqueuses prélevées sur les animaux adaptés

\begin{tabular}{|c|c|c|c|c|}
\hline & $\begin{array}{l}30 \text { min avant } \\
\text { injection }\end{array}$ & 30 min après $\left({ }^{1}\right)$ & $120 \underset{\text { min après }}{\left({ }^{1}\right)}$ & $\begin{array}{l}\text { Signification } \\
\text { statistique }\end{array}$ \\
\hline $\mathrm{NaCl} 0,9$ p. 100 (A) & $100(2257 \pm 290)$ a $\left(2^{2} 4\right)$ & $95,4 \pm 1,4 \mathrm{~b} * *$ & $96,2 \pm 1,6 b *$ & $\begin{aligned} * P & <0,05 \\
* * P & <0,001\end{aligned}$ \\
\hline $\begin{array}{l}\text { Glucose } 15 \text { p. } 100 \text { (B) } \\
\text { Muqueuse amidon }\left({ }^{3}\right)\end{array}$ & $\begin{array}{l}100(2056 \pm 854) a \\
100(1830+177) a\end{array}$ & $\begin{array}{c}84,8 \pm 2,5 b * * \\
(5) P<0,01 \\
133,6 \pm 3,9 b\end{array}$ & $\begin{array}{c}88,2 \pm 3,2 b * \\
(5) P<0,01 \\
134,4+4,1 b\end{array}$ & $\begin{aligned} * P & <0,01 \\
* * P & <0,001 \\
P & <0,001\end{aligned}$ \\
\hline $\begin{array}{l}30 \text { min (C) } \\
\text { Muqueuse amidon }\left({ }^{3}\right) \\
60 \text { min (D) }\end{array}$ & $100(1586 \pm 410) a$ & $\begin{array}{l}\left(^{5}\right) \mathrm{P}<0,001 \\
136,6 \pm 5,6 \mathrm{~b} \\
\left(^{5}\right) \mathrm{P}<0,001 \\
\left.{ }^{6}\right) \mathrm{NS}\end{array}$ & $\begin{array}{l}(5) P<0,001 \\
136,6 \pm 2,5 b \\
\left({ }^{5}\right) P<0,001 \\
\left(^{(6)} \mathrm{NS}\right.\end{array}$ & $P<0,001$ \\
\hline
\end{tabular}

Activité spécifique de l'amylase (unités/mg protéines tołales) (cf. légende tabl. 1).

Le traitement pratiqué avec les lyophilisats de muqueuse induit pendant toute la durée de l'éłude une augmentation significative de l'activité spécifique de l'amylase de l'ordre de 40 p. 100 . L'effet est semblable quel que soit le temps de prélèvement de la muqueuse après le repas.

En ce qui concerne l'activité spécifique de la lipase (tabl. 4) les données présenfées montrent que l'injection intraveineuse de la solution de glucose 15 p. 100 ne modifie pas l'activité spécifique de l'enzyme, tant en comparaison avec celle observée après injection de $\mathrm{NaCl}$ 0,9 p. 100 que par rapport à la valeur basale. Le même traitement

\section{TABLEAU 4}

Effets sur l'octivité spécifique de la lipase dans le suc pancréatique des injections intraveineuses de $\mathrm{NaCl}$, de glucose ef des lyophilisots de muqueuses prélevées sur les animaux adaptés

\begin{tabular}{|c|c|c|c|c|}
\hline & $\begin{array}{l}30 \text { min avant } \\
\text { injection }\end{array}$ & $\begin{array}{c}30 \text { min après }\left(^{1}\right) \\
\text { injection }\end{array}$ & $120 \min _{\text {injection }}\left({ }^{1}\right)$ & $\begin{array}{l}\text { Signification } \\
\text { statistique }\end{array}$ \\
\hline $\begin{array}{l}\mathrm{NaCl} 0,9 \text { p. } 100 \text { (A) } \\
\text { Glucose } 15 \text { p. } 100 \text { (B) }\end{array}$ & $\begin{array}{l}100(36,1 \pm 3,6) a\left({ }^{2,4}\right) \\
100(34,1 \pm 4,2) a\end{array}$ & $\begin{array}{l}98,8 \pm 0,9 a \\
99,6 \pm 3 a \\
(5) \mathrm{NS}\end{array}$ & $\begin{array}{l}98,6 \pm 1,8 a \\
100,8 \pm 1,5 a \\
(5) \mathrm{NS}\end{array}$ & $\begin{array}{l}\text { NS } \\
\text { NS }\end{array}$ \\
\hline $\begin{array}{l}\text { Muqueuse amidon }\left({ }^{3}\right) \\
30 \text { min }(C) \\
\text { Muqueuse amidon }\left({ }^{3}\right) \\
60 \text { min (D) }\end{array}$ & $\begin{array}{l}100(35+5,2) a \\
100(36,8+5,2) a\end{array}$ & $\begin{array}{c}91,8 \pm 1,9 b^{*} \\
(5) P<0,01 \\
86,2 \pm 3,3 b^{* *} \\
\left(^{5}\right) \mathrm{P}<0,01 \\
\text { (6) NS }\end{array}$ & $\begin{array}{c}90,8 \pm 0,9 b^{* *} \\
(5) P<0,05 \\
88 \pm 3,2 b^{*} \\
\left(^{5}\right) \mathrm{P}<0,025 \\
(6) \mathrm{NS}\end{array}$ & $\begin{aligned} * P & <0,005 \\
* * P & <0,001 \\
* P & <0,01 \\
P & <0,005\end{aligned}$ \\
\hline
\end{tabular}

Activité spécifique de la lipase (unités/mg protéines totales) (cf. légende tabl. 1). 
pratiqué avec les lyophilisats de muqueuse duodénale diminue significativement l'activité spécifique de cette enzyme. La diminution de l'activité spécifique de la lipase est légèrement plus impartante avec la muqueuse prélevée $60 \mathrm{~min}$ après le début de la prise du repas qu'avec celle prélevée $30 \mathrm{~min}$ après, mais la différence n'est pas statistiquement significative.

Les résultats relatifs à l'activité spécifique de la chymoirypsine (tabl. 5) montrent que l'injection intraveineuse de la solution de glucose 15 p. 100 provoque une augmentation peu importante mais statistiquement significative de sa valeur. La diminution de l'activité spécifique de la chymotrypsine, observée après l'injection intraveineuse des lyophilisats de muqueuse est peu prononcée ef n'est significative que pour la muqueuse prélevée 60 min après le début de la prise du repas et pour la période $120 \mathrm{~min}$. D'une manière générale, les valeurs enregistrées après injection intraveineuse des lyophilisats ne sont pas différentes de celles observées après injection de la solution isotonique de chlorure de sodium.

\section{TABLEAU 5}

Effets sur l'activité spécifique de la chymotrypsine dans le suc pancréatique des injections intraveineuses de $\mathrm{NaCl}$, de glucose et des lyophilisats de muqueuses prélevées sur les animaux adaptés

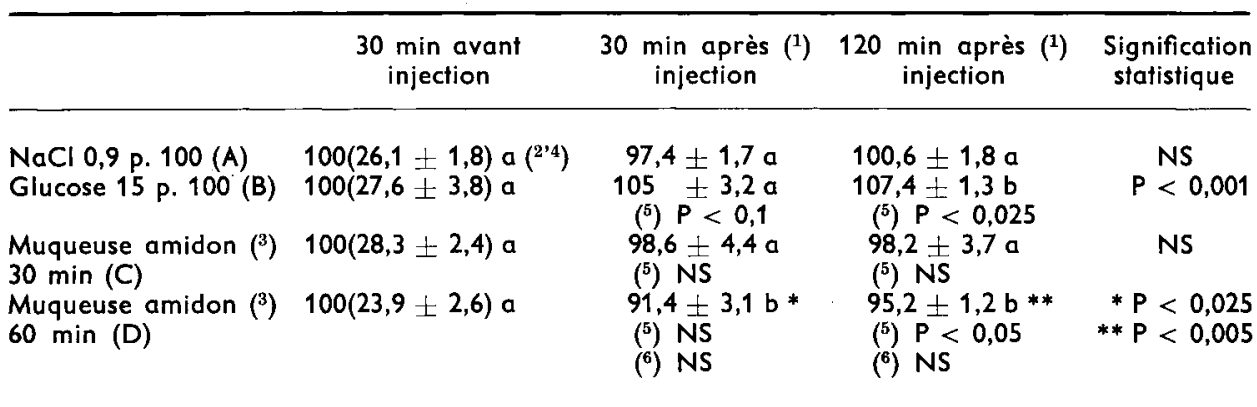

Activité spécifique de la chymotrypsine (unités/mg protéines tolales) (cf. légende tabl. 1).

\section{Discussion.}

L'évolution des caractéristiques de la sécrétion pancréatique exocrine a été étudiée en réponse à l'injection de différents produits.

L'injection intraveineuse de glucose en solution hypertonique (15 p. 100) augmente très légèrement l'activité spécifique de la chymotrypsine, n'affecte pas celle de la lipase et inhibe le volume, les protéines totales excrétées ainsi que l'activité spécifique de l'amylase. Cette inhibition confirme des observations antérieures chez le Porc (Simoes Nunes et Corring, 1979b), chez l'Homme (Dudrick et al., 1970) et chez le Chien (Nakajima et Magee, 1970 ; Saito et al., 1978). D'autre part, Saito et al. (1978) ont montré que la pression osmotique des solutions employées (glucose 20 p. 100 ef 30 p. 100) n'était pas responsable des effets observés. Ces résultats apportent un argument en faveur de l'absence d'intervention du glucose après absorption (entendue ici comme le passage dans la circulation sanguine) et à court terme dans l'adap- 
tation de l'amylase pancréatique au régime alimentaire. Par contre, chez le Chien, selon Crider et al. (1956) l'injection intraveineuse de glucose augmente la teneur en amylase tant dans le tissu que dans le suc pancréatique excrété. De même, chez le Rat, l'administration à long terme de glucose par voie intrapéritonéale (Morisset ef Dunningan, 1971) ou intraveineuse (Bazin, 1978) provoque une augmentation de l'activité spécifique de l'amylase du tissu pancréatique. En ce qui concerne l'effet sur les activités spécifiques de la chymotrypsine et de la lipase les résultats de Nakajima et Magee (1970) sont opposés aux nôtres dans la mesure où ils ont observé une diminution des activités spécifiques des deux enzymes. Toutefois, ces auteurs ont aussi constaté que la chute de l'activité était plus importante pour l'amylase que pour la chymotrypsine ef la lipase. Aucune autre étude, à notre connaissance, n'a comporté l'analyse des activités de ces dernières enzymes.

L'augmentation du volume de suc excrété à la suite de l'injection intraveineuse des lyophilisats de muqueuse duodénale confirme l'observation de Hickson (1970). Cet effet ainsi que l'augmentation des protéines totales excrétées sont très probablement la résultante de la présence dans les lyophilisats de sécrétine ef de cholecystokinine-pancréozymine (CCK-PZ). Ces deux hormones sont en effet stimulatrices de la sécrétion hydroélectrolytique (sécrétine) et enzymatique (CCK-PZ) du pancréas (Johnson, 1977).

L'injection intraveineuse des lyophilisats de muqueuse duodénale provenant d'animaux adaptés à un régime riche en amidon provoque une augmentation importante de l'activité spécifique de l'amylase et une relative inhibition de l'activité spécifique de la lipase. Ces effets sont très semblables avec les deux lyophilisats employés. Le glucose a été trouvé à l'état de traces dans les deux lyophilisats et de ce fait ne peut être considéré dans l'induction des modifications observées des activités spécifiques des enzymes. On peut plus vraisemblablement penser à des phénomènes provoqués au niveau de la muqueuse duodénale par la variation des quantités de produits d'hydrolyse digestive présents dans le duodénum. Selon certains auteurs (Felber, Zermatten et Dick, 1974 ; Dick et Felber, 1975), les produits d'hydrolyse digestive agiraient sur la synthèse et/ou la libération par la muqueuse duodénale de principes de nature hormonale responsables de l'excrétion spécifique accrue de certaines enzymes pancréatiques. Toutefois, Bode, Dürr et Bode (1979) et La Bella et al. (1979) n'ont pas pu confirmer l'hypothèse de Félber, Zermatten et Dick (1974). Mais Adelson ef Rothman (1974) ont isolé de la muqueuse duodénale du Porc un facteur, la chymodénine, susceptible de produire une sécrétion préférentielle de chymotrypsinogène après injection par voie intraveineuse. Grossman (1974) classe la chymodenine comme «un peptide pur de la muqueuse intestinale avec statut hormonal incertain ».

Cependant, les variations quantitatives des produits de sécrétion ef d'hydrolyse gastrique arrivant au duodénum et induites par les modifications de la composition du régime alimentaire peuvent provoquer la libération accrue de sécrétine ou de CCK-PZ. En effet, on sait d'une part que chacune de ces deux hormones est plus ou moins stimulée selon le principe présent dans le duodénum (Wang et Grossman, 1951) ef d'autre part que la stimulation du pancréas exocrine par la CCK-PZ associée à la sécrétine conduit à une sécrétion non parallèle d'enzymes (Dagorn et Mongeau, 1977 ; Rinderknecht et al., 1978). Il nous semble que l'hypothèse d'une libération différentielle de CCK-PZ, de sécrétine et éventuellement des peptides intestinaux à action 
de type «incretine » à la suite de modifications de la composition alimentaire ne doif pas être a priori écartée dans la recherche des mécanismes possibles de l'adaptation de la sécrétion pancréatique exocrine au régime alimentaire. Cette possibilité ainsi que celle concernant l'isolement, la purification et la vérification des effets physiologiques des composants duodénaux hypothétiques synthétisés et/ou libérés par la présence de quantités importantes de produits d'hydrolyse digestive restent à étayer ef aussi à vérifier.

Journées Ingestion-Digestion-Absorption de l'Association française de Nutrition, Poris, 15-16 novembre 1979.

\section{Références}

ADELSON J., ROTHMAN S., 1974. Selective pancreatic enzyme secretion due to a new peptide called chymodenin. Science, 183, 1087-1089.

BAZIN R., 1978. Adaptation du pancréas exocrine d̀ la composition du régime alimentaire chez le rat. Th., Etat (Paris VI), 29-32.

BEN ABDELJLIL A., DESNUELLE P., 1964. Sur l'adaptation des enzymes exocrines du pancréas à la composition du régime. Bioch. biophys. Acta, 81, 136-149.

BODE C., DÜRR H. K., BODE J. C., 1979. On the existence of specific duodenal hormones for the regulation of pancreatic enzyme secretion following injection of certain food substracts (glucose and protein hydrolysat). Gastroent. clin. biol., 3, 300-301.

CORRING T., 1974. Régulation de la sécrétion pancréatique par rétro-inhibition négative chez le porc. Ann. Biol. anim. Bioch. Biophys., 12, 233-241.

CORRING T., 1977. Possible role of hydrolysis products of the dietary components in the mechanisms of the exocrine pancreatic adaptation to the diet. WId. Rev. Nutr. Diet., 27, 132-144.

CORRING T., AUMAITRE A., RÉRAT A., 1972. Fistulation permanente du pancréas exocrine chez le Porc. Application : influence du repas. Ann. Biol. anim. Bioch. Biophys., 12, 109-124.

CRIDER J. O., CONLY S. S., DORCHESTER J. E. C., THOMAS J. E., 1956. Effect of intravenous injection of hypertonic glucose solution on external secretion of the pancreas. Am. J. Physiol., 186, 187-189.

DAGORN J. C., MONGEAU R., 1977. Different action of hormonal stimulation on the biosynthesis of three pancreatic enzymes. Bioch. biophys. Acta, 498, 76-82.

DICK J., FELBER J. P., 1975. Specific hormonal regulation by food on the pancreas (amylase and trypsin) secretions. Horm. Metab. Res., 7, 161-166.

DUDRICK S. J., WILMORE D. W., STEIGER E., MACKIE J. A., FITTS W. J., 1970. Spontaneous closure of traumatic pancreato-duodenal fistulas with total intravenous nutrition. J. Trauma., 10, 542-543.

FELBER J. P., ZERMATTEN A., DICK J., 1974. Modulation by food, of hormonal system regulating rat pancreatic secretion. Lancet, 2, 185-187.

GROSSMAN M. I., 1974. Candidate hormones of the gut. Gastroenterology, 67, 730-755.

HICKSON J. C. D., 1970. The secretory and vascular response to nervous and hormonal stimulation in the pancreas of the pig. J. Physiol., 206, 299-322.

HUGGETT A. S. G., NIXON D. A., 1957. Enzymatic defermination of blood glucose. Biochem. J., 66, 12 pp.

JOHNSON A., HURWITZ R., KRETCHMER N., 1977. Adaptation of rat pancreatic amylase and chymotrypsinogen to changes in diet. J. Nuir., 107, 87-96.

JOHNSON L. R., 1977. Gastro-intestinal hormones and their functions. Ann. Rev. Physiol., 39, 135-158.

LA BELLA A., DAGORN J. C., FIGARELLA C., SARLES H., 1979 . Lack of specific stimulation of pancreatic enzymes by the related nutriments. Gastroent. clin. biol., 3, 301.

MORISSET J., DUNNINGAN J., 1971. Effects of glucose, amino acids and insulin on adaptation of exocrine pancreas to diet. Proc. Soc. exp. Biol. Med., 136, 231-234.

NAKAJIMA S., MAGEE D. F., 1970. Inhibition of exocrine pancreatic secretion by glucagon and D-glucose given intravenously. Canad. J. Physiol. Pharmac., 48, 299-305. 
RINDERKNECHT H., RENNER I. G., DOUGLAS A. P., ADHAM N. F., 1978. Profiles of pure pancreatic secretions obtained by direct pancreatic duct cannulation in normal healthy human subjects. Gasfroenterology, 75, 1083-1089.

SAITO Y., TOKUTAKE S., MATSUNO S., NOTO N., HONDA T., SATO T., 1978. Effects of hypertonic glucose and amino acid infusions on pancreatic exocrine function. Tohuku J. exp. Med., 124, 99-115.

SIMCES NUNES C., CORRING T., 1979a. Pancreatic exocrine secretion in the pig following test meals of different composition and intra-duodenal loads of glucose and maltose. Horm. Mefab. Res., 11, 346-351.

SIMCES NUNES C., CORRING T., 1979b. Données non publiées.

SNOOK J. T., 1971. Dietary regulation of pancreatic enzymes with emphasis on carbohydrate. Am. J. Physiol., 221, 1383-1387.

WANG C. C., GROSSMAN M. I., 1951. Physiological determination of release of secretin and pancreozymin from intestine of dogs with transplanted pancreas. Am. J. Physiol., 164, 527-545. 